Алгебра и анализ

Том. 16 (2004), вып. 5
St. Petersburg Math. J.

Vol. 16 (2005), No. 5, Pages 773-790 S 1061-0022(05)00878-2

Article electronically published on September 21, 2005

\title{
ON SPECTRUM GAPS \\ OF SOME DIVERGENT ELLIPTIC OPERATORS WITH PERIODIC COEFFICIENTS
}

\author{
V. V. ZHIKOV \\ Dedicated to Mikhail Shlyomovich Birman on the occasion of his anniversary
}

\section{$\S 1$. InTRODUCTION}

More than 10 years ago physicists gave a theoretic description of the so-called photonic crystal, an optic analog of a semiconductor. In contrast to a semiconductor, the photonic crystal is an artificial material, a composite. The dominant requirement for the photonic crystal is that electromagnetic waves of a certain length cannot propagate in it. It was also predicted that the photonic crystal is a material with high-contrast periodic structure [1]. In the mathematical sense, here we have a periodic Maxwell operator in the entire space $L^{2}\left(\mathbb{R}^{d}\right)$, and this operator must have gaps in its spectrum. Since the Maxwell operator is quite difficult from the viewpoint of spectral theory, scalar second-order elliptic operators ("acoustic approximations") are often considered.

There are many mathematical publications on this subject, in which different methods are applied depending on what specific geometric and physical model of the photonic crystal is chosen. For a detailed statement of the problem and a review of mathematical methods and models, see the paper [2] by Figotin and Kuchment and the papers [3, 4] by Kuchment and Kunyansky.

1. We recall the description of the spectrum of an operator with periodic coefficients. Let

$$
A=-\operatorname{div}(a \nabla)=-\nabla^{*}(a \nabla),
$$

where the coefficient $a=a(x)$ is measurable and periodic,

$$
a(x+n)=a(x), \quad n \in Z^{d},
$$

and satisfies the following condition of boundedness and ellipticity:

$$
0<\alpha \leq a(x) \leq \alpha^{-1} .
$$

We consider a family of problems with quasiperiodic conditions on the boundary of the periodicity cell $\square=[0,1)^{d}$, namely,

$$
\begin{gathered}
-\operatorname{div}(a \nabla u)=\lambda u, \\
u(x)=e^{i k \cdot x} v(x), \quad v \in H_{\mathrm{per}}^{1}(\square),
\end{gathered}
$$

2000 Mathematics Subject Classification. Primary 35P15.

Key words and phrases. Photonic crystal, periodic Maxwell operator, spectral gap.

This work was supported by RFBR (grant no. 02-01-00114), and by the Ministry of Education (Fundamental Research grant no. 02-1.0-57). 
where $k \in \mathbb{R}^{d}$ is a quasimomentum and $H_{\text {per }}^{1}(\square)$ is the Sobolev space of periodic functions. Then we have the following periodic problem for $v(x)$ :

$$
A(k) v=\lambda v, \quad A(k)=-(\nabla+i k)^{*} a(\nabla+i k) .
$$

By definition, the function $v \in H_{\text {per }}^{1}(\square)$ is a solution of this problem if the following integral identity is satisfied:

$$
\int_{\square} a(\nabla v+i k v) \cdot(\nabla \bar{\varphi}-i k \bar{\varphi}) d x=\lambda \int_{\square} v \bar{\varphi} d x, \quad \varphi \in H_{\mathrm{per}}^{1}(\square),
$$

where the bar means complex conjugation. Putting $\varphi=v$, we obtain

$$
\int_{\square} a|\nabla u|^{2} d x=\lambda \int_{\square}|u|^{2} d x
$$

Each operator $A(k)$ is selfadjoint in $L^{2}(\square)$ and has a compact resolvent. We order the eigenvalues of the operator $A(k)$ in accordance with the minimax principle, i.e.,

$$
0 \leq E_{1}(k) \leq E_{2}(k) \leq \cdots .
$$

The band functions $E_{n}(k)$ are continuous and $2 \pi$-periodic with respect to $k \in \mathbb{R}^{d}$, and the spectrum of $A$ is the union of the segments (bands) that are the images of the band functions

$$
\operatorname{Sp} A=\bigcup\left[\alpha_{n}, \beta_{n}\right], \quad \alpha_{n}=\min _{k} E_{n}, \quad \beta_{n}=\max _{k} E_{n} .
$$

Successive segments $\left[\alpha_{n}, \beta_{n}\right]$ and $\left[\alpha_{n+1}, \beta_{n+1}\right]$ may overlap, but if they are disjoint, then we have a gap in the spectrum.

The existence of gaps in the spectrum is of interest from a physical viewpoint; this is related to wave propagation. Consider the wave equation

$$
u_{t t}^{\prime \prime}-A u=f_{0} e^{i \omega t}, \quad f_{0} \in L^{2}\left(\mathbb{R}^{d}\right),
$$

the solution of which is

$$
u(t)=u_{0} e^{i \omega t}, \quad u_{0}=-\left(A+\omega^{2} I\right)^{-1} f_{0} .
$$

If $\omega^{2}$ is in a spectral gap, then $u_{0} \in L^{2}\left(\mathbb{R}^{d}\right)$, and the wave is localized. In experiments, the amplitude $f_{0}$ is very small and the wave $u(t)$ is also small and usually cannot be observed (is perceived as identically zero). However, if the number $\omega^{2}$ belongs to the spectrum, then the amplitude $u_{0}$ is not localized and can take considerably large values depending on the location of the point $\omega^{2}$ in $\operatorname{Sp} A$.

The presence of gaps in the spectrum is the main characteristic property of the "photonic crystal". Recently, this subject has attracted considerable interest of physicists and mathematicians.

In the present paper, we discuss a method for the study of spectral gaps. This method is based on averaging theory and was suggested in the paper [5] without any association with photonic crystals.

2. First, we restrict ourselves to the simplest geometric model studied in [5, 6]. In [6], Hempel and Lienau considered the operator

$$
A^{t}=-\operatorname{div}\left(a^{t}(y) \nabla\right), \quad t \rightarrow \infty,
$$

where the coefficient $a^{t}$ has period 1 and is defined by

$$
a^{t}(y)= \begin{cases}1 & \text { on the periodic disperse set } F_{0}, \\ t^{2} & \text { outside of } F_{0}\end{cases}
$$

(see Figure 1, where the periodicity cell is shown by a dashed line).

They proved that, as $t \rightarrow \infty$, the operator $A^{t}$ has at least one gap in its spectrum; this paper contains many other valuable observations. 


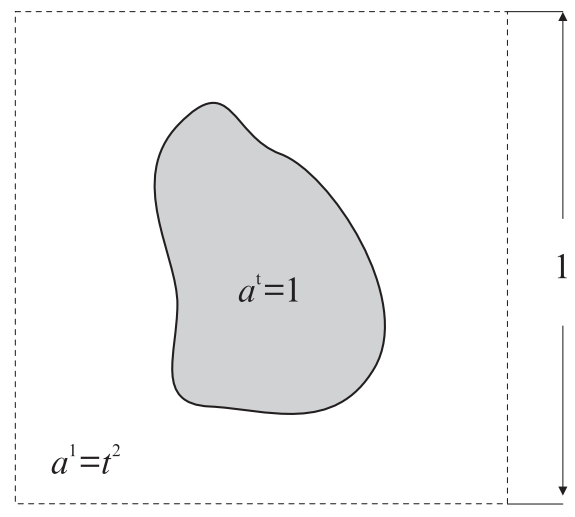

FIGURE 1.

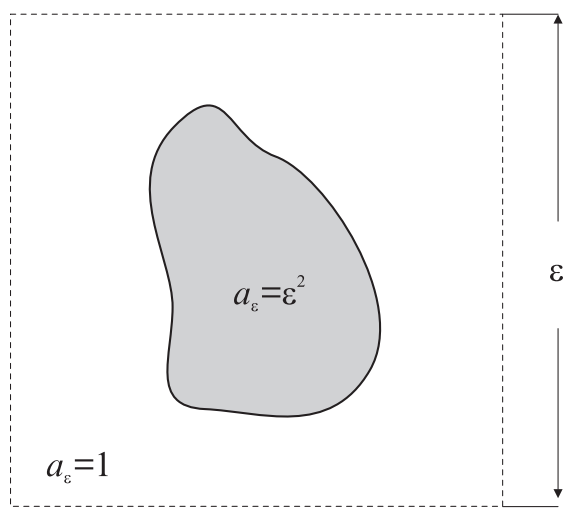

FIGURE 2.

In [5], the present author considered the operator

$$
A_{\varepsilon}=-\operatorname{div}\left(a_{\varepsilon}(x) \nabla\right)
$$

where the coefficient $a_{\varepsilon}(x)$ has period $\varepsilon$ and is defined as follows:

$$
a_{\varepsilon}(x)= \begin{cases}\varepsilon^{2} & \text { on } F_{0}^{\varepsilon} \text { (soft phase), } \\ 1 & \text { on } \mathbb{R}^{d} \backslash F_{0}^{\varepsilon} \text { (rigid phase), }\end{cases}
$$

where $F_{0}^{\varepsilon}=\varepsilon F_{0}=\left\{\varepsilon x, x \in F_{0}\right\}$ is a homothetic contraction of the disperse set $F_{0}$ (Figure 2). The operator $A_{\varepsilon}$ corresponds to the double-porosity model.

It was proved that, as $\varepsilon \rightarrow 0$, the operator $A_{\varepsilon}$ has gaps in the spectrum, and that the number of gaps increases unboundedly as $\varepsilon \rightarrow 0$.

It can easily be seen that the spectra of $A^{t}$ and $A_{\varepsilon}$ coincide. Indeed, if $\lambda \in \operatorname{Sp} A^{t}$ and

$$
-\operatorname{div}_{y}\left(a^{t}(y) \nabla_{y} u\right)=\lambda u(y)
$$

then, using the change of variables $y=\varepsilon^{-1} x$, we obtain

$$
-\operatorname{div}\left(a_{\varepsilon}(x) \nabla u(x)\right)=\lambda u(x),
$$

so that

$$
\operatorname{Sp} A^{t}=\operatorname{Sp} A_{\varepsilon} \quad \text { for } t=\varepsilon^{-1} .
$$

This is the only common feature of the operators $A^{t}$ and $A_{\varepsilon}$; in all other respects they differ considerably. For example, the operator $A^{t}$ is bounded from below by the operator 
$-\triangle$ (because $a^{t} \geq 1$ ). Therefore, the density of states is bounded as $t \rightarrow \infty$; the corresponding limit was found in [6], see also [7]. On the contrary, the operator $A_{\varepsilon}$ is unbounded from below, and the density of states is unbounded as $\varepsilon \rightarrow 0$. The methods used in the two papers mentioned above are entirely different.

We prefer to operate with real function spaces and real solutions of elliptic equations; only the Bloch eigenfunctions are regarded as complex solutions.

\section{§2. Resolvent CONVERGEnCE}

1. Many homogenization problems are of the form

$$
A_{\varepsilon} u_{\varepsilon}+s u_{\varepsilon}=f
$$

where $s>0$, and the $A_{\varepsilon}$ are nonnegative selfadjoint operators in the Hilbert space $H$, $f \in H$. The result itself of homogenization means the strong convergence $u_{\varepsilon} \rightarrow u$ and the identity

$$
A u+s u=f
$$

where $A$ is also a nonnegative selfadjoint operator, which is said to be a homogenized or a limit operator. This situation corresponds to the so-called strong resolvent convergence

$$
\left(A_{\varepsilon}+s\right)^{-1} f \rightarrow(A+s)^{-1} f
$$

for all $f \in H$ and all $s>0$ (it suffices to have this for $s=1$ ).

As an example, we can take the operator

$$
A_{\varepsilon}=-\operatorname{div}\left(a\left(\frac{x}{\varepsilon}\right) \nabla\right), \quad H=L^{2}\left(\mathbb{R}^{d}\right),
$$

where the coefficient $a(y)$ is periodic and measurable and satisfies condition (1.1). Then homogenization theory gives the strong convergence

$$
\left(A_{\varepsilon}+1\right)^{-1} f \rightarrow(A+1)^{-1} f, \quad f \in L^{2}\left(\mathbb{R}^{d}\right), \quad A=-\operatorname{div}\left(\mathbf{a}^{\text {hom }} \nabla\right),
$$

where $\mathbf{a}^{\text {hom }}$ is a constant positive definite matrix.

Recently, Birman and Suslina ([8]; see also [18]) refined this result by proving the convergence in norm and the estimate

$$
\left\|\left(A_{\varepsilon}+1\right)^{-1}-(A+1)^{-1}\right\| \leq c \varepsilon .
$$

For the operator $A_{\varepsilon}$ with the coefficient $a_{\varepsilon}$ defined by (1.4), nothing of this kind can be stated. Since the coefficient $a_{\varepsilon}$ is asymptotically degenerate, the family $u_{\varepsilon}$ of solutions of the resolvent equation

$$
u_{\varepsilon} \in H^{1}\left(\mathbb{R}^{d}\right), \quad-\operatorname{div}\left(a_{\varepsilon} \nabla u_{\varepsilon}\right)+u_{\varepsilon}=f \in L^{2}\left(\mathbb{R}^{d}\right)
$$

is not compact in $L^{2}\left(\mathbb{R}^{d}\right)$ and even in $L^{2}(\Omega)$, where $\Omega$ is a bounded region.

In this case, strong convergence is out of the question. The family $u_{\varepsilon}$ is bounded in $L^{2}\left(\mathbb{R}^{d}\right)$, and we can try to find an equation satisfied by the weak limit $\lim _{\varepsilon \rightarrow 0} u_{\varepsilon}$. However, this way is not efficient since weak convergence has many pathologies; in particular, the limit equation can lose its resolvent character.

As was shown in [5], the sequence $u_{\varepsilon}$ is compact in the sense of the so-called strong twoscale convergence. The two-scale limit of the sequence of solutions $u_{\varepsilon}$ is not a function in $L^{2}\left(\mathbb{R}^{d}\right)$, but a function $u=u(x, y)$ of two variables periodic in $y$ and belonging to $L^{2}\left(\mathbb{R}^{d} \times \square\right)$. In accordance with this, the limit operator is defined not in $L^{2}\left(\mathbb{R}^{d}\right)$ but in a wider Hilbert space $H \subset L^{2}\left(\mathbb{R}^{d} \times \square\right)$.

2. We give the definition of two-scale convergence (see [9]). First, we recall the mean value property of a periodic function. 
The mean value property. Let $\Phi(x)$ be a periodic function defined on $\mathbb{R}^{d}$, and let $\Phi \in L_{\text {per }}^{1}(\square)$ and $\langle\Phi\rangle=\int_{\square} \Phi d y$. Then, for each $\varphi \in C_{0}^{\infty}\left(\mathbb{R}^{d}\right)$ we have

$$
\lim _{\varepsilon \rightarrow 0} \int_{\mathbb{R}^{d}} \varphi(x) \Phi\left(\frac{x}{\varepsilon}\right) d x=\langle\Phi\rangle \int_{\mathbb{R}^{d}} \varphi(x) d x .
$$

Let $\Omega$ be an arbitrary region in $\mathbb{R}^{d}$, e.g., $\Omega=\mathbb{R}^{d}$. We say that a sequence $v_{\varepsilon}$ bounded in $L^{2}(\Omega)$ is weakly two-scale convergent to a function $v \in L^{2}(\Omega \times \square), v_{\varepsilon}(x) \stackrel{2}{\rightarrow} v(x, y)$, if

$$
\lim _{\varepsilon \rightarrow 0} \int_{\Omega} v_{\varepsilon}(x) \varphi(x) b\left(\frac{x}{\varepsilon}\right) d x=\int_{\square} \int_{\Omega} v(x, y) \varphi(x) b(y) d x d y
$$

for all $\varphi \in C_{0}^{\infty}(\Omega)$ and all $b \in C_{\mathrm{per}}^{\infty}(\square)$.

Example. If $f \in C_{0}^{\infty}(\Omega)$ and $\Phi \in L_{\text {per }}^{2}(\square)$, then $v_{\varepsilon}(x)=f(x) \Phi\left(\frac{x}{\varepsilon}\right) \stackrel{2}{\rightarrow} f(x) \Phi(y)$. This follows from the mean value property.

We say that a sequence $u_{\varepsilon}$ bounded in $L^{2}(\Omega)$ is strongly two-scale convergent to a function $u \in L^{2}(\Omega \times \square), u_{\varepsilon}(x) \stackrel{2}{\longrightarrow} u(x, y)$, if

$$
\lim _{\varepsilon \rightarrow 0} \int_{\Omega} u_{\varepsilon}(x) v_{\varepsilon}(x) d x=\int_{\square} \int_{\Omega} u(x, y) v(x, y) d x d y \quad \text { if } v_{\varepsilon}(x) \stackrel{2}{\rightarrow} v(x, y) .
$$

We list some properties of two-scale convergence:

(i) a sequence bounded in $L^{2}(\Omega)$ is compact in the sense of weak two-scale convergence;

(ii) if $v_{\varepsilon}(x) \stackrel{2}{\rightarrow} v(x, y)$, then

$$
\liminf _{\varepsilon \rightarrow 0} \int_{\Omega}\left|v_{\varepsilon}\right|^{2} d x \geq \int_{\Omega} \int_{\square}|v|^{2} d x d y ;
$$

(iii) $v_{\varepsilon}(x) \stackrel{2}{\longrightarrow} v(x, y)$ if and only if $v_{\varepsilon}(x) \stackrel{2}{\rightarrow} v(x, y)$ and

$$
\lim _{\varepsilon \rightarrow 0} \int_{\Omega}\left|v_{\varepsilon}\right|^{2} d x=\int_{\Omega} \int_{\square}|v|^{2} d x d y ;
$$

(iv) if $f_{\varepsilon}(x) \rightarrow f(x)$ in $L^{2}(\Omega)$, then $f_{\varepsilon}(x) \stackrel{2}{\rightarrow} f(x)$;

(v) if $f \in C_{0}^{\infty}(\Omega)$ and $b \in L_{\text {per }}^{2}(\square)$, then $f(x) b\left(\frac{x}{\varepsilon}\right) \stackrel{2}{\longrightarrow} f(x) b(y)$;

(vi) for every $f \in L^{2}(\Omega \times \square)$ there is a family $f_{\varepsilon} \in C_{0}^{\infty}(\Omega)$ such that $f_{\varepsilon} \stackrel{2}{\longrightarrow} f$.

Now, we give the corresponding generalization of the strong resolvent convergence.

Let $H$ be a subspace of $L^{2}\left(\mathbb{R}^{d} \times \square\right)$, and let $P: L^{2}\left(\mathbb{R}^{d} \times \square\right) \rightarrow H$ be the orthogonal projection.

Definition 2.1. If $A_{\varepsilon}$ and $A$ are nonnegative selfadjoint operators in $L^{2}\left(\mathbb{R}^{d}\right)$ and in $H$, respectively, then the strong two-scale resolvent convergence $A_{\varepsilon} \stackrel{2}{\longrightarrow} A$ means that

$$
\left(A_{\varepsilon}+1\right)^{-1} f_{\varepsilon} \stackrel{2}{\longrightarrow}(A+1)^{-1} P f \quad \text { if } f_{\varepsilon} \stackrel{2}{\longrightarrow} f, f \in L^{2}\left(\mathbb{R}^{d} \times \square\right) .
$$

To study the spectrum of the operator $A_{\varepsilon}$ for small $\varepsilon$, it is necessary to know the spectrum of the limit operator A, together with some facts concerning the "convergence" of the spectra of $A_{\varepsilon}$ to the spectrum of $A$.

The convergence of the spectra in the sense of Hausdorff is most desired. By definition, this means that

(i) for all $\lambda \in \operatorname{Sp} A$ there are $\lambda_{\varepsilon} \in \operatorname{Sp} A_{\varepsilon}$ such that $\lambda_{\varepsilon} \rightarrow \lambda$;

(ii) if $\lambda_{\varepsilon} \in \operatorname{Sp} A_{\varepsilon}$ and $\lambda_{\varepsilon} \rightarrow \lambda$, then $\lambda \in \operatorname{Sp} A$.

Proposition 2.2. Property (i) is always valid under the strong two-scale resolvent convergence. 
Proof. We put $T_{\varepsilon}=\left(A_{\varepsilon}+1\right)^{-1}$ and $T=(A+1)^{-1}$.

If $\lambda \in \operatorname{Sp} A$, then $\mu=(1+\lambda)^{-1} \in \operatorname{Sp} T$, and, therefore, for each $\delta>0$ there is an element $f$ in $H$ such that

$$
\|f\|_{H}=1, \quad\|T f-\mu f\|_{H} \leq \frac{\delta}{4} .
$$

We take $f_{\varepsilon} \in L^{2}\left(\mathbb{R}^{d}\right)$ so that $f_{\varepsilon} \stackrel{2}{\longrightarrow} f$, and, in particular, $\lim _{\varepsilon \rightarrow 0}\left\|f_{\varepsilon}\right\|_{L^{2}\left(\mathbb{R}^{d}\right)}=\|f\|_{H}$ (see (2.4)). Then, by the resolvent convergence (2.5), we have

$$
\lim _{\varepsilon \rightarrow 0}\left\|\left(T_{\varepsilon}-\mu\right) f_{\varepsilon}\right\|_{L^{2}\left(\mathbb{R}^{d}\right)}=\|(T-\mu) f\|_{H} \leq \frac{\delta}{4} .
$$

We obtain $\left\|\left(T_{\varepsilon}-\mu\right) f_{\varepsilon}\right\|_{L^{2}\left(\mathbb{R}^{d}\right)} \leq \delta / 2,\left\|f_{\varepsilon}\right\|_{L^{2}\left(\mathbb{R}^{d}\right)} \geq 1 / 2$ for sufficiently small $\varepsilon$. It is well known that this implies that the interval $(-\delta+\mu, \mu+\delta)$ contains a point of the spectrum of $T_{\varepsilon}$. We see that every interval centered at $\lambda$ contains points of the spectrum of $A_{\varepsilon}$ if $\varepsilon$ is sufficiently small. The proposition is proved.

Resolvent convergence implies the convergence of spectral projections. We consider the spectral expansions of the operators $A_{\varepsilon}$ and $A$,

$$
A_{\varepsilon}=\int_{0}^{\infty} \lambda d E_{\varepsilon}(\lambda) \text { in } L^{2}\left(\mathbb{R}^{d}\right), \quad A=\int_{0}^{\infty} \lambda d E(\lambda) \text { in } H .
$$

Proposition 2.3. If $\lambda$ is not an eigenvalue of the operator $A$, then

$$
E_{\varepsilon}(\lambda) f_{\varepsilon} \stackrel{2}{\longrightarrow} E(\lambda) f \quad \text { whenever } f_{\varepsilon} \stackrel{2}{\longrightarrow} f, f \in H .
$$

We do not dwell on the proof of this statement, which, of course, implies the Hausdorff convergence property (i). Property (ii) is subtler; mostly, it does not occur under resolvent convergence.

Now, we can describe our approach to the problem of gaps in spectra.

Let $A_{\varepsilon}$ be the operator of double porosity (1.3), (1.4).

Proposition 2.4. We have the two-scale resolvent convergence $A_{\varepsilon} \stackrel{2}{\longrightarrow} A$.

This result was proved in [5] and is quite general. Here, it does not matter what the soft phase is: it can be a dispersed set or a structure of three-dimensional lattice type. It is only required that the rigid phase $\mathbb{R}^{d} \backslash F_{0}$ be connected.

Proposition 2.5. The spectrum of the limit operator has infinitely many gaps.

We prove this for a dispersed $F_{0}$. In the case of a structure of three-dimensional lattice type, this is also true, but the description of gaps looks somewhat differently.

Proposition 2.6. If the soft phase is dispersed, then property (ii) of the Hausdorff convergence of spectra is satisfied.

This is a key point, and the dispersity of the soft phase is essential here.

It remains to use the following quite obvious statement.

Proposition 2.7. Let $K_{\varepsilon}$ and $K$ be closed sets on the real line, and suppose we have the Hausdorff convergence $K_{\varepsilon} \rightarrow K$. If the limit set $K$ has infinitely many gaps, then, for sufficiently small $\varepsilon$, the set $K_{\varepsilon}$ has gaps close to gaps in $K$, and the number of gaps grows unboundedly as $\varepsilon \rightarrow 0$.

Hempel and Lienau proved also that, as $t \rightarrow \infty$, the spectrum of $A^{t}$ converges in the sense of Hausdorff to a closed set that has at least one gap. However, they did not identify this limit set with the spectrum of an operator. 
We make some remarks concerning resolvent convergence, which, however, will not be used directly.

For our purposes, it suffices to have the strong convergence (2.5) only for $f \in H$, and then the projection $P$ can be dropped. However, in homogenization problems, convergence with projection occurs, and it is important that this strong convergence is equivalent to a certain special weak convergence of operators.

Proposition 2.8. Let $T_{\varepsilon}$ and $T$ be bounded selfadjoint operators in $L^{2}\left(\mathbb{R}^{d}\right)$ and $\left.L^{2}\left(\mathbb{R}^{d} \times \square\right)\right)$, respectively, and let $\left\|T_{\varepsilon}\right\| \leq 1$ and $\|T\| \leq 1$. Then the convergence

$$
T_{\varepsilon} f_{\varepsilon} \stackrel{2}{\longrightarrow} T f \quad \text { whenever } f_{\varepsilon} \stackrel{2}{\longrightarrow} f
$$

is equivalent to the convergence

$$
T_{\varepsilon} g_{\varepsilon} \stackrel{2}{\rightarrow} T g \quad \text { whenever } g_{\varepsilon} \stackrel{2}{\rightarrow} g
$$

Proof. $1^{\circ}$. Suppose (2.7) is valid. Since $T_{\varepsilon} g_{\varepsilon}$ is bounded in $L^{2}\left(\mathbb{R}^{d}\right)$, we may assume that $T_{\varepsilon} g_{\varepsilon} \stackrel{2}{\rightarrow} z$, and we must prove that $z=T g$. We have

$$
\int_{\mathbb{R}^{d}} T_{\varepsilon} g_{\varepsilon} f_{\varepsilon} d x \rightarrow \int_{\mathbb{R}^{d}} \int_{\square} z \cdot f d x d y
$$

because $f_{\varepsilon} \stackrel{2}{\longrightarrow} f$. On the other hand,

$$
\int_{\mathbb{R}^{d}} T_{\varepsilon} g_{\varepsilon} \cdot f_{\varepsilon} d x=\int_{\mathbb{R}_{\varepsilon}} g_{\varepsilon} T_{\varepsilon} f_{\varepsilon} d x \rightarrow \int_{\square} \int_{\mathbb{R}^{d}} g \cdot T f d x d y=\int_{\square} \int_{\mathbb{R}^{d}} T g \cdot f d x d y
$$

by (2.7). Consequently, $z=T g$.

$2^{\circ}$. Suppose $(2.8)$ is valid. Then

$$
\int_{\mathbb{R}^{d}} T_{\varepsilon} f_{\varepsilon} \cdot g_{\varepsilon} d x=\int_{\mathbb{R}^{d}} f_{\varepsilon} \cdot T_{\varepsilon} g_{\varepsilon} d x \rightarrow \int_{\square} \int_{\mathbb{R}^{d}} f \cdot T g d x d y=\int_{\square} \int_{\mathbb{R}^{d}} T f \cdot g d x d y .
$$

Since the sequence $g_{\varepsilon}$ with $g_{\varepsilon} \stackrel{2}{\longrightarrow} g$ is arbitrary, we have $T_{\varepsilon} f_{\varepsilon} \rightarrow T f$ by the definition of strong convergence (see (2.3)). The proposition is proved.

Thus, the strong convergence of the resolvents (2.5) is equivalent to the convergence

$$
\left(A_{\varepsilon}+1\right)^{-1} f_{\varepsilon} \stackrel{2}{\rightarrow}(A+1)^{-1} P f \quad \text { whenever } f_{\varepsilon} \stackrel{2}{\rightarrow} f, f \in L^{2}\left(\mathbb{R}^{d} \times \square\right) .
$$

In homogenization theory, this "weak convergence" is proved first, and then the "strong convergence" (2.5) is deduced from it.

It should be noted that the convergence (2.5) does not imply a similar convergence for spectral projections, i.e., we cannot replace $f$ by $P f$ in (2.6) and assume that $f$ is an arbitrary element of $L^{2}\left(\mathbb{R}^{d} \times \square\right)$. The same is true for the Trotter-Kato theorem: for $t \geq 0$ we have

$$
e^{-t A_{\varepsilon}} f_{\varepsilon} \stackrel{2}{\longrightarrow} e^{-t A} f \quad \text { whenever } f_{\varepsilon} \stackrel{2}{\longrightarrow} f, f \in H .
$$

We cannot find an equivalent "weak" statement for this strong operator convergence.

\section{§3. The LIMIT OpERATOR}

We consider the set $V$ of functions of the form

$$
\begin{aligned}
& u(x, y)=u_{1}(x)+u_{0}(x, y), \quad u_{1} \in H^{1}\left(\mathbb{R}^{d}\right), \\
& u_{0} \in L^{2}\left(\mathbb{R}^{d}, H_{\mathrm{per}}^{1}(\square)\right),\left.\quad u_{0}(x, \cdot)\right|_{\mathbb{R}^{d} \backslash F_{0}}=0 .
\end{aligned}
$$

For clarity, we restrict ourselves to the case where the soft phase $F_{0}$ is dispersed. Let $B=F_{0} \cap \square$ be the soft insertion in the periodicity cell. We assume that the boundary 
of $B$ is Lipschitz. Then the component $u_{0}(x, \cdot)$ is a function of class $H_{0}^{1}(B)$, extended by zero to $\square \backslash B$, and we have

$$
V=H^{1}\left(\mathbb{R}^{d}\right)+L^{2}\left(\mathbb{R}^{d}, H_{0}^{1}(B)\right) .
$$

In the Hilbert space $L^{2}\left(\mathbb{R}^{d} \times \square\right)=L^{2}\left(\mathbb{R}^{d}, L^{2}(\square)\right)$, we consider the subspace

$$
H=L^{2}\left(\mathbb{R}^{d}\right)+L^{2}\left(\mathbb{R}^{d}, L^{2}(B)\right),
$$

which is closed because if $f(x, y)=f_{1}(x)+f_{0}(x, y)$, where $f_{1} \in L^{2}\left(\mathbb{R}^{d}\right)$ and $f_{0} \in$ $L^{2}\left(\mathbb{R}^{d}, L^{2}(B)\right)$, then

$$
\int_{\square} \int_{\mathbb{R}^{d}}|f|^{2} d x d y \geq\left(1-|B|^{1 / 2}\right)\left[\int_{\mathbb{R}^{d}}\left|f_{1}\right|^{2} d x+\int_{B} \int_{\mathbb{R}^{d}}\left|f_{0}\right|^{2} d x d y\right] .
$$

It is also obvious that the set $V$ is dense in $H$.

On the set $V$, we define the quadratic form

$$
Q(u, u)=\int_{\mathbb{R}^{d}} \mathbf{a}^{\text {hom }} \nabla u_{1} \cdot \nabla \bar{u}_{1} d x+\int_{B} \int_{\mathbb{R}^{d}} \nabla_{y} u_{0} \cdot \nabla_{y} \bar{u}_{0} d x d y,
$$

where $\mathbf{a}^{\text {hom }}$ is the homogenized matrix

$$
\mathbf{a}^{\text {hom }} \xi \cdot \xi=\inf _{w \in C_{\text {per }}^{\infty}(\square)} \int_{\square \backslash B}|\xi+\nabla w|^{2} d y, \quad \xi \in \mathbb{R}^{d},
$$

which is positive definite, because the rigid phase $\mathbb{R}^{d} \backslash F_{0}$ is connected in $\mathbb{R}^{d}$.

Inequality (3.2) implies that the form $Q$ is closed. Therefore, this form determines a nonnegative selfadjoint operator $A$ in $H$. The equation $A u=P f$, where $f \in L^{2}\left(\mathbb{R}^{d} \times \square\right)$ and $P: L^{2}\left(\mathbb{R}^{d} \times \square\right) \rightarrow H$ is an orthogonal projection, means that the following integral identity is valid:

$$
\int_{\mathbb{R}^{d}} \mathbf{a}^{\text {hom }} \nabla u_{1} \cdot \nabla \varphi_{1} d x+\int_{B} \int_{\mathbb{R}^{d}} \nabla_{y} u_{0} \cdot \nabla_{y} \varphi_{0} d x d y=\int_{\square} \int_{\mathbb{R}^{d}} f \varphi d x d y
$$

for every test function $\varphi=\varphi_{1}+\varphi_{0} \in V$. Putting $\varphi_{0}=0$ in the above identity, and then $\varphi_{1}=0$, we obtain the following two relations:

$$
\left\{\begin{aligned}
-\operatorname{div}\left(\mathbf{a}^{\text {hom }} \nabla u_{1}\right) & =\langle f\rangle & & \text { in } \mathbb{R}^{d}, \\
-\triangle_{y} u_{0} & =f & & \text { in } \mathbb{R}^{d} \times \square .
\end{aligned}\right.
$$

For the projection $P: L^{2}\left(\mathbb{R}^{d} \times \square\right) \rightarrow H$, we have the relation

$$
P f=g(x, y)= \begin{cases}f(x, y) & \text { if } y \in F_{0}, \\ \int_{\square \backslash B} f(x, z) d z & \text { if } y \in \mathbb{R}^{d} \backslash F_{0} .\end{cases}
$$

Obviously, if we replace $f$ by $P f$, we do not change equations (3.4). We represent these equations in a shorter form:

$$
\begin{cases}A_{1} u_{1}=\langle f\rangle & \text { in } L^{2}\left(\mathbb{R}^{d}\right) \text { (space operator) } \\ A_{0} u_{0}=f & \text { in } L^{2}\left(\mathbb{R}^{d}, L^{2}(B)\right) \text { (Bloch operator). }\end{cases}
$$

The "space operator" $A_{1}=-\operatorname{div}\left(\mathbf{a}^{\text {hom }} \nabla\right)$ is an elliptic second-order operator with constant coefficients. Regarded as an operator in $L^{2}\left(\mathbb{R}^{d}\right)$, it has an absolutely continuous spectrum that fills the entire nonnegative axis. The Bloch operator $A_{0}$ reduces to the Laplace-Dirichlet operator $-\triangle_{y}$ in $L^{2}(B)$ (we preserve the same notation $A_{0}$ for this operator). Below, we prove that the spectrum of $A$ (including the gaps in it) is determined by the Bloch operator $A_{0}$. 


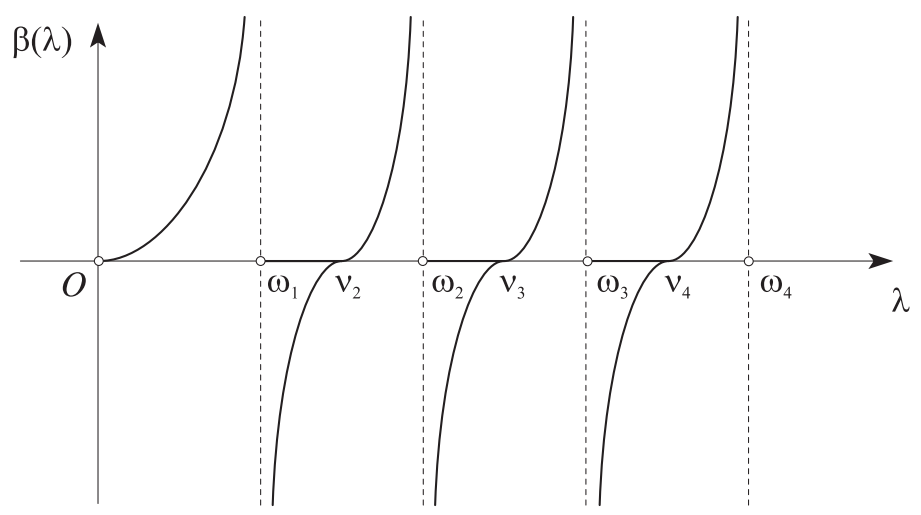

FiguRE 3.

We consider the following eigenvalue problem:

$$
\begin{gathered}
A u=\lambda u, \quad u=u_{1}+u_{0}, \\
\left\{\begin{array}{l}
A_{0} u_{0}=\lambda\left(u_{1}+u_{0}\right), \\
A_{1} u_{1}=\lambda\left\langle u_{1}+u_{0}\right\rangle .
\end{array}\right.
\end{gathered}
$$

We note that if $u_{0}$ is an eigenfunction of the Laplace-Dirichlet operator, $A_{0} u_{0}=\lambda u_{0}$, and $\left\langle u_{0}\right\rangle=0$, then $u(x, y)=u_{0}(y)$ is an eigenfunction of $A$. Obviously, for $l \in L^{2}\left(\mathbb{R}^{d}\right)$ the function $l(x) u_{0}(y)$ is also an eigenfunction. These are purely Bloch eigenfunctions, and, as shown below, they exhaust the point spectrum of $A$.

The Laplace-Dirichlet operator $A_{0}$ has discrete spectrum. We split this spectrum into two disjoint parts:

$$
\text { Sp } A_{0}=\left\{\omega_{1}, \omega_{2}, \ldots\right\} \cup\left\{\omega_{1}^{\prime}, \omega_{2}^{\prime}, \ldots\right\} .
$$

The second part consists of the eigenvalues for which all corresponding eigenfunctions have zero mean. We consider the expansion

$$
1=\sum_{n=1}^{\infty} c_{n} \varphi_{n}, \quad c_{n}=\left\langle\varphi_{n}\right\rangle \neq 0
$$

where the $\varphi_{n}$ are the eigenfunctions of $A_{0}, A_{0} \varphi_{n}=\omega_{n} \varphi_{n}$, normalized in $L^{2}(B)$. Then

$$
b=b(y, \lambda)=\sum_{n=1}^{\infty} \frac{c_{n} \varphi_{n}(y)}{\omega_{n}-\lambda}
$$

is a solution of the equation

$$
A_{0} b=\lambda b+1, \quad \lambda \notin\left\{\omega_{1}, \omega_{2}, \ldots\right\} .
$$

We put

$$
\beta(\lambda)=\lambda(1+\lambda\langle b\rangle)=\lambda+\sum_{n=1}^{\infty} \frac{c_{n}^{2} \lambda^{2}}{\omega_{n}-\lambda}
$$

Lemma 3.1. All eigenfunctions of the operator $A$ are among the purely Bloch eigenfunctions mentioned above.

Proof. Let $u=u_{1}+u_{0}$ be an eigenfunction of $A$ (see (3.6)). If $u_{1} \equiv 0$, then $\left\langle u_{0}\right\rangle=0$. Therefore, $u=u_{0}$ is a purely Bloch eigenfunction. We prove that the relation $u_{1} \equiv 0$ is always valid. 
Let $u_{1} \not \equiv 0$. We consider the orthogonal expansion

$$
u_{0}=\sum v_{n}(x) \varphi_{n}(y)+\sum v_{n}^{\prime}(x) \varphi_{n}^{\prime}(y),
$$

where the $\varphi_{n}$ and $\varphi_{n}^{\prime}$ are the eigenfunctions of $A_{0}$ corresponding to the eigenvalues in $\left\{\omega_{1}, \omega_{2}, \ldots\right\}$ and in $\left\{\omega_{1}^{\prime}, \omega_{2}^{\prime}, \ldots\right\}$, respectively. By (3.6), we have

$$
\begin{gathered}
\left(A_{0}-\lambda\right) u_{0}=\lambda u_{1}, \\
\sum\left(\omega_{n}-\lambda\right) v_{n} \varphi_{n}+\sum\left(\omega_{n}^{\prime}-\lambda\right) v_{n}^{\prime} \varphi_{n}^{\prime}=\lambda u_{1} \sum c_{n} \varphi_{n} .
\end{gathered}
$$

It follows that $\lambda \notin\left\{\omega_{1}, \omega_{2}, \ldots\right\}$, because

$$
\left(\omega_{n}-\lambda\right) v_{n}=\lambda u_{1} c_{n} \neq 0,
$$

and if $\lambda=\omega_{k}^{\prime}$, then

$$
u_{0}(x, y)=\lambda u_{1}(x) \sum_{n=1}^{\infty} \frac{c_{n} \varphi_{n}(y)}{\omega_{n}-\lambda}+v_{k}^{\prime}(x) \varphi_{k}^{\prime}(y) .
$$

Since $\left\langle\varphi_{k}^{\prime}\right\rangle=0$, we have

$$
\lambda\left\langle u_{1}+u_{0}\right\rangle=\beta(\lambda) u_{1}(x),
$$

and (3.6) implies the relation $A_{1} u_{1}=\beta(\lambda) u_{1}$, from which it is clear that $u_{1} \equiv 0$, because the operator $A_{1}$ has no eigenfunctions in $L^{2}\left(\mathbb{R}^{d}\right)$. The lemma is proved.

The graph of $\beta(\lambda)$ is shown in Figure 3. The function $\beta$ strictly increases on the intervals between the points $0, \omega_{1}, \omega_{2}, \ldots$.

Lemma 3.2. $A$ point $\lambda$ belongs to the resolvent set of $A$ if $\beta(\lambda)<0$ and $\lambda \notin \operatorname{Sp} A_{0}$.

Proof. We must prove that the problem

$$
\left\{\begin{array}{l}
A_{0} u_{0}-\lambda\left(u_{1}+u_{0}\right)=f \\
A_{1} u_{1}-\lambda\left\langle u_{1}+u_{0}\right\rangle=\langle f\rangle
\end{array}\right.
$$

has a solution for every $f \in H$ provided $\lambda$ satisfies the assumptions of the lemma. Since $\lambda \notin \operatorname{Sp} A_{0}$, we can put

$$
u_{0}=g+\lambda u_{1} b,
$$

where $g=\left(A_{0}-\lambda\right)^{-1} f$ and $b$ is a solution of (3.9). Hence,

$$
A_{1} u_{1}-\beta(\lambda) u_{1}=\langle f\rangle+\lambda\langle g\rangle .
$$

It remains to observe that, by the inequality $\beta(\lambda)<0$, the operator $A_{1}-\beta(\lambda) I$ is invertible in $L^{2}\left(\mathbb{R}^{d}\right)$ and equation (3.12) is solvable. Consequently, problem (3.11) has a solution for every $f \in H$. The lemma is proved.

Lemma 3.3. The segments on which $\beta \geq 0$ belong to the spectrum of $A$.

Proof. Assuming the contrary, we find a point $\lambda$ lying inside one of the segments indicated and belonging to the resolvent set of $A$. Then problem (3.11) is solvable for every $f \in H$. We consider this problem in the specific case where $f=\langle f\rangle$. It is easy to realize that $\lambda \notin \operatorname{Sp} A_{0}$. Therefore, (3.11) implies

$$
\begin{aligned}
u_{0} & =\left(\lambda u_{1}+f\right) b, \\
A_{1} u_{1}-\beta(\lambda) u_{1} & =f(1+\lambda\langle b\rangle) .
\end{aligned}
$$

Since $(1+\lambda\langle b\rangle)=\lambda^{-1} \beta(\lambda)>0$, we see that the equation $A_{1} u_{1}-\beta(\lambda) u_{1}=g$ has a solution in $L^{2}\left(\mathbb{R}^{d}\right)$ for every $g \in L^{2}\left(\mathbb{R}^{d}\right)$, which is impossible because $\beta(\lambda)>0$. The lemma is proved. 
We describe the zeros $0=\nu_{1}<\nu_{2}<\nu_{3}<\cdots$ of $\beta(\lambda)$ (see Figure 3) with the help of the so-called electrostatic problem. On the set $\mathbb{C}^{1}+H_{0}^{1}(B)$, we consider the quadratic form

$$
Q(u, u)=\int_{B} \nabla u_{0} \cdot \nabla \bar{u}_{0} d y, \quad u=t+u_{0}, \quad t \in \mathbb{C}^{1}, u_{0} \in H_{0}^{1}(B),
$$

and study the spectrum of the corresponding selfadjoint operator $\Gamma$ in the Hilbert space $\mathbb{C}^{1}+L^{2}(B)$ regarded as a subspace of $L^{2}(\square)$. By definition, the equation

$$
\Gamma u=\lambda u, \quad u=t+u_{0},
$$

means that

$$
\int_{B} \nabla \varphi_{0} \cdot \nabla \varphi_{0} d y=\lambda \int_{\square}\left(t+u_{0}\right)\left(c+\varphi_{0}\right) d y, \quad c \in \mathbb{C}^{1}, \varphi_{0} \in H_{0}^{1}(B) .
$$

For $\lambda=0$, the eigenfunction $u \equiv t$ is constant. For $\lambda>0,(3.13)$ implies that $t=-\left\langle u_{0}\right\rangle$ and

$$
-\triangle u_{0}=\lambda\left(u_{0}-\left\langle u_{0}\right\rangle\right) \quad \text { in } B .
$$

If $\left\langle u_{0}\right\rangle=0$, then $u=u_{0}$ is an eigenfunction of $A_{0}$ with eigenvalue $\lambda \in\left\{\omega_{1}^{\prime}, \omega_{2}^{\prime}, \ldots\right\}$. This trivial part of the spectrum of the electrostatic problem belongs also to the spectrum of the Dirichlet problem. In the case where $\left\langle u_{0}\right\rangle \neq 0$, we necessarily have $\lambda \notin\left\{\omega_{1}, \omega_{2}, \ldots\right\}$. Indeed, if $\lambda=\omega_{n}$, then the equation $-\Delta \varphi_{n}=\omega_{n} \varphi_{n}$ and (3.14) imply

$$
\begin{aligned}
& \int_{B} \nabla u_{0} \cdot \nabla \varphi_{n} d y=\omega_{n} \int_{B}\left(u_{0}-\left\langle u_{0}\right\rangle\right) \varphi_{n} d y, \\
& \int_{B} \nabla \varphi_{n} \cdot \nabla u_{0} d y=\omega_{n} \int_{B} \varphi_{n} u_{0} d y .
\end{aligned}
$$

Therefore, $\omega_{n}\left\langle u_{0}\right\rangle\left\langle\varphi_{n}\right\rangle=0$. Since $\omega_{n}>0$ and $\left\langle\varphi_{n}\right\rangle \neq 0$, we have $\left\langle u_{0}\right\rangle=0$.

Thus, $\lambda \notin\left\{\omega_{1}, \omega_{2}, \ldots\right\}$. Then equation (3.14) can easily be solved,

$$
u_{0}(y)=-\lambda\left\langle u_{0}\right\rangle b(y, \lambda),
$$

and we see that

$$
\left\langle u_{0}\right\rangle(1+\lambda\langle b\rangle)=0 \Longrightarrow \beta(\lambda)=0 .
$$

Conversely, if $\beta(\lambda)=0$, then the function $u_{0}=-\lambda b(y, \lambda)$ satisfies the equation

$$
-\Delta u_{0}=\lambda\left(u_{0}-1\right) \quad \text { in } B,
$$

and $\left\langle u_{0}\right\rangle=1$, i.e., $u_{0}-1$ is an eigenfunction of the operator $\Gamma$. Thus, we have proved the following statement.

Lemma 3.4. The zeros of $\beta(\lambda)$ are nontrivial eigenvalues of the electrostatic problem (i.e., they are not eigenvalues of the Dirichlet problem).

Now, we can describe the spectrum of the operator $A$.

1. Some eigenvalues of the Laplace-Dirichlet operator $A_{0}$ (namely, those for which the corresponding eigenfunctions have zero mean) are eigenvalues (of infinite multiplicity) of the operator $A$. The operator $A$ does not have other eigenvalues. Thus, the point spectrum of $A$ contains the points $\omega_{1}^{\prime}, \omega_{2}^{\prime}, \ldots$ (see (3.7)) and also all multiple eigenvalues in $\left\{\omega_{1}, \omega_{2}, \ldots\right\}$. It is well known (see [10]) that, for a "typical" region $B$, the spectrum of the Laplace-Dirichlet operator is simple and the eigenfunctions have zero mean; therefore, the set $\left\{\omega_{1}^{\prime}, \omega_{2}^{\prime}, \ldots\right\}$ is empty. In this case, $A$ has no eigenvalues.

2. Consider the restriction of $A$ to the orthogonal complement of the set of all its eigenfunctions. The spectrum of this restriction is the union of the segments on which $\beta \geq 0$, and the intervals between them are gaps. It can easily be proved that the 
spectrum is absolutely continuous inside the latter intervals, and the "eigenfunctions of the continuous spectrum" look like this:

$$
u(x, y, \lambda)=e^{i p \cdot x}\{1+\lambda b(y, \lambda)\}, \quad \mathbf{a}^{\text {hom }} p \cdot p=\beta(\lambda) .
$$

3. The interval $\left(\omega_{i}, \nu_{i+1}\right)(i=1,2, \ldots)$ is not necessarily a gap because it can contain an eigenvalue among $\left\{\omega_{1}^{\prime}, \omega_{2}^{\prime}, \ldots\right\}$. Then this interval splits into several gaps. In any case, the spectrum of $A$ contains infinitely many gaps.

Similar results are valid in the case where the scalar conductivity coefficient $a_{\varepsilon}(x)$ (see (1.4)) is replaced by the matrix coefficient

$$
\mathbf{a}_{\varepsilon}(x)= \begin{cases}\varepsilon^{2} \mathbf{a}\left(\frac{x}{\varepsilon}\right) & \text { on } F_{0}^{\varepsilon} \text { (soft phase) } \\ \mathbf{a}\left(\frac{x}{\varepsilon}\right) & \text { on } \mathbb{R}^{d} \backslash F_{0}^{\varepsilon} \text { (rigid phase), }\end{cases}
$$

where $\mathbf{a}(y)$ is a measurable periodic symmetric matrix satisfying the usual conditions of boundedness and ellipticity,

$$
\alpha \xi^{2} \leq \mathbf{a} \xi \cdot \xi \leq \alpha^{-1} \xi^{2}, \quad \alpha>0 .
$$

The homogenized matrix $\mathbf{a}^{\text {hom }}$ is defined by the equation

$$
\mathbf{a}^{\text {hom }} \xi \cdot \xi=\inf _{w \in C_{\text {per }(\square)}^{\infty}} \int_{\square \backslash B} \mathbf{a}(y)(\xi+\nabla w) \cdot(\xi+\nabla \bar{w}) d y,
$$

and for the role of $A_{0}$ we must take the operator $-\operatorname{div}_{y}\left(\mathbf{a}(y) \nabla_{y}\right)$ corresponding to the Dirichlet problem in the region $B$.

\section{§4. Convergence of SPECTRA}

1. Here we prove the missing property (ii) of the Hausdorff convergence of spectra. Let $\lambda_{\varepsilon} \in \operatorname{Sp} A_{\varepsilon}$ be such that $\lambda_{\varepsilon} \rightarrow \lambda$; we must prove that $\lambda \in \operatorname{Sp} A$. Since we know that $\operatorname{Sp} A_{0} \subset \operatorname{Sp} A$, we assume that $\lambda \notin \operatorname{Sp} A_{0}$. We have

$$
A_{\varepsilon} u_{\varepsilon}=\lambda_{\varepsilon} u_{\varepsilon}
$$

where the eigenfunction $u_{\varepsilon}$ is quasiperiodic on the cell $\varepsilon \square$ and satisfies the normalization condition

Then (see (1.2))

$$
f_{\varepsilon \square}\left|u_{\varepsilon}\right|^{2} d x=\frac{1}{\varepsilon^{d}} \int_{\varepsilon \square}\left|u_{\varepsilon}\right|^{2} d x=1 .
$$

$$
f_{\varepsilon \square} a_{\varepsilon}\left|\nabla u_{\varepsilon}\right|^{2} d x=\lambda_{\varepsilon}
$$

and for each cube $\Omega=[-t, t]^{d}, t \geq 1$, we have

$$
\begin{aligned}
& \frac{1}{2} \leq f_{\Omega}\left|u_{\varepsilon}\right|^{2} d x \leq 2, \\
& f_{\Omega} a_{\varepsilon}\left|\nabla u_{\varepsilon}\right|^{2} d x \leq 2 \lambda_{\varepsilon},
\end{aligned}
$$

provided $\varepsilon$ is sufficiently small.

Homogenization in double-porosity models was studied in the papers [10]-[13] and [5]. We need the following result.

Theorem 4.1. Let $\Omega$ be a closed Lipschitz region, and let $u_{\varepsilon} \in H^{1}(\Omega)$ be a sequence such that

1) $\lim \sup _{\varepsilon \rightarrow 0} \int_{\Omega}\left(\left|u_{\varepsilon}\right|^{2}+a_{\varepsilon}\left|\nabla u_{\varepsilon}\right|^{2}\right) d x<\infty$;

$2)-\operatorname{div}\left(a_{\varepsilon} \nabla u_{\varepsilon}\right)=g_{\varepsilon}, g_{\varepsilon}$ is bounded in $L^{2}(\Omega), g_{\varepsilon}(x) \stackrel{2}{\rightarrow} g(x, y)$. 
Then (up to extraction of a subsequence) we have the two-scale convergence

$$
u_{\varepsilon}(x) \stackrel{2}{\rightarrow} u(x, y)=u_{1}(x)+u_{0}(x, y), \quad u_{1} \in H^{1}(\Omega), \quad u_{0} \in L^{2}\left(\Omega, H_{0}^{1}(B)\right),
$$

and the limit function satisfies the integral identity

$$
\int_{\Omega} \mathbf{a}^{\text {hom }} \nabla u_{1} \cdot \nabla \varphi_{1} d x+\int_{\Omega} \int_{B} \nabla_{y} u_{0} \cdot \nabla_{y} \varphi_{0} d x d y=\int_{\Omega} \int_{\square} g\left(\varphi_{1}+\varphi_{0}\right) d x d y
$$

for all $\varphi_{1} \in C_{0}^{\infty}(\Omega)$ and all $\varphi_{0} \in L^{2}\left(\Omega, H_{0}^{1}(B)\right)$.

In a concise form, we can write

$$
\begin{cases}A_{1} u_{1}=-\operatorname{div}\left(\mathbf{a}^{\text {hom }} \nabla u_{1}\right)=\langle g\rangle & \text { in } \Omega, \\ A_{0} u_{0}=-\Delta_{y} u_{0}=g & \text { in } \Omega \times \square .\end{cases}
$$

Applying Theorem 4.1 to the sequence of eigenfunctions $u_{\varepsilon}$, we obtain the two-scale convergence

$$
u_{\varepsilon}(x) \stackrel{2}{\rightarrow} u(x, y)=u_{1}(x)+u_{0}(x, y), \quad u_{1} \in H_{\mathrm{loc}}^{1}\left(\mathbb{R}^{d}\right), \quad u_{0} \in L_{\mathrm{loc}}^{2}\left(\mathbb{R}^{d}, H_{0}^{1}(B)\right),
$$

and equations (3.6). Formally, the equation $A u=\lambda u$ is fulfilled. However, the function $u$ is not an element of $L^{2}\left(\mathbb{R}^{d} \times \square\right)$, and, above all, it is unclear whether $u \neq \equiv 0$.

The following statement plays a key role.

Lemma 4.2 (compactness lemma). Let $\lambda_{\varepsilon} \rightarrow \lambda \notin \operatorname{Sp} A_{0}$. Then the sequence of eigenfunctions $u_{\varepsilon}$ is compact in the sense of strong two-scale convergence in every bounded region $\Omega \subset \mathbb{R}^{d}$.

Using this lemma, inequality (4.1), and property (2.4), we obtain $f_{\Omega \times \square}|u|^{2} d x d y \geq$ $1 / 2$. Then also $u_{1} \neq 0$ because otherwise (3.6) implies $\lambda \in \operatorname{Sp} A_{0}$. Now, property (ii) of the Hausdorff convergence can easily be obtained. Indeed, by (3.6), we have the following equation for $u_{1}$ :

$$
-\operatorname{div}\left(\mathbf{a}^{\text {hom }} \nabla u_{1}\right)=\beta(\lambda) u_{1},
$$

and if we assume that $\lambda \notin \operatorname{Sp} A$, then $\beta(\lambda)<0$ by Lemma 3.3. Relation (4.1) and property (2.3) of semicontinuity yield

$$
f_{\Omega} \int_{\square}|u|^{2} d x d y \leq 2
$$

Now, an inequality of the form (3.2) (with $\Omega$ instead of $\mathbb{R}^{d}$ ) leads to the estimate

$$
f_{\Omega}\left|u_{1}\right|^{2} d x \leq \frac{2}{1-|B|^{1 / 2}}
$$

which shows that the solution $u_{1}$ is of "moderate" growth, i.e., it corresponds to a "tempered distribution" (a continuous functional on the Schwartz space on $\mathbb{R}^{d}$ ). Then $u_{1} \equiv 0$, which becomes clear if we pass to the Fourier transform in (4.2) and recall that $\beta(\lambda)<0$.

2. To prove the compactness lemma, we need the following well-known result concerning extension of functions (see [14, Chapter III]).

Proposition 4.3. Suppose $B_{1}$ is a sufficiently smooth region such that $\bar{B}_{1} \subset \square, \bar{B} \subset B_{1}$, and $B_{1} \backslash B$ is connected. Then there is an extension of $u \in H^{1}\left(B_{1} \backslash B\right)$ up to a function $\tilde{u} \in H^{1}\left(B_{1}\right)$, and we have the estimates

$$
\int_{B}|\nabla \tilde{u}|^{2} d x \leq C \int_{B_{1} \backslash B}|\nabla u|^{2} d x, \quad \int_{B}|\tilde{u}|^{2} d x \leq C \int_{B_{1} \backslash B}\left(|u|^{2}+|\nabla u|^{2}\right) d x
$$


with a constant $C$ independent of $u$. Moreover, we may assume that the function $\tilde{u}$ is harmonic in $B$.

Estimates (4.3) only improve under a homothety, and we can apply them to each component of $F_{0}^{\varepsilon}$. As a result, we obtain an extension of $\left.u_{\varepsilon}\right|_{\mathbb{R}^{d} \backslash F_{0}^{\varepsilon}}$ to $\mathbb{R}^{d}$,

$$
\tilde{u}_{\varepsilon} \in H_{\mathrm{loc}}^{1}\left(\mathbb{R}^{d}\right), \quad f_{\Omega}\left(\left|\tilde{u}_{\varepsilon}\right|^{2}+\left|\nabla \tilde{u}_{\varepsilon}\right|^{2}\right) d x \leq C_{1} .
$$

Without loss of generality, we may assume that

$$
\tilde{u_{\varepsilon}} \rightarrow u_{1} \quad \text { in } H_{\mathrm{loc}}^{1}\left(\mathbb{R}^{d}\right) .
$$

Since $\tilde{u}_{\varepsilon} \rightarrow u_{1}$ strongly in $L^{2}(\Omega)$, we have $\tilde{u}_{\varepsilon}(x) \stackrel{2}{\rightarrow} u(x)$ (see property (iv) of two-scale convergence in $\S 2$ ). Therefore, it remains to prove that the difference $v_{\varepsilon}=u_{\varepsilon}-\tilde{u}_{\varepsilon}$ is compact in the sense of strong two-scale convergence in $\Omega$. Assuming that $\tilde{u}_{\varepsilon}$ is harmonic on each inclusion, we obtain

$$
v_{\varepsilon} \in H_{0}^{1}\left(F_{0}^{\varepsilon}\right), \quad-\varepsilon^{2} \triangle v_{\varepsilon}=\lambda_{\varepsilon}\left(\tilde{u}_{\varepsilon}+v_{\varepsilon}\right) \text { in } F_{0}^{\varepsilon} .
$$

Consider the operator $T_{\varepsilon}=-\varepsilon^{2} \Delta$ corresponding to the Dirichlet problem in $F_{0}^{\varepsilon}$. Since $F_{0}^{\varepsilon}$ splits into separate components, the operator $T_{\varepsilon}$ also splits, and its spectrum coincides with that of the operator $A_{0}$ in $L^{2}(B)$. Since the points $\lambda_{\varepsilon}$ are separated away from $\operatorname{Sp} A_{0}$ by a distance $\rho_{0}>0$, we obtain $\left\|\left(T_{\varepsilon}-\lambda_{\varepsilon}\right)^{-1}\right\| \leq \frac{1}{\rho_{0}}$, and for the solution of the equation

$$
T_{\varepsilon} z_{\varepsilon}-\lambda_{\varepsilon} z_{\varepsilon}=g_{\varepsilon}
$$

we have the estimate

$$
\left\|z_{\varepsilon}\right\|_{L^{2}\left(\Omega \cap F_{0}^{\varepsilon}\right)} \leq \frac{1}{\rho_{0}}\left\|g_{\varepsilon}\right\|_{L^{2}\left(\Omega \cap F_{0}^{\varepsilon}\right)},
$$

where $\Omega \cap F_{0}^{\varepsilon}$ means the totality of inclusions entirely lying in $\Omega$.

Below, we use the following obvious property of two-scale convergence.

Proposition 4.4. Suppose $v_{\varepsilon} \in L^{2}(\Omega)$ and $v \in L^{2}(\Omega \times \square)$. Assume that the following condition is fulfilled: for every $\delta>0$ there exist elements $z_{\varepsilon} \in L^{2}(\Omega)$ and $z \in L^{2}(\Omega \times \square)$ such that

$$
z_{\varepsilon} \stackrel{2}{\longrightarrow} z, \quad\|v-z\|_{L^{2}(\Omega \times \square)} \leq \delta, \quad \limsup _{\varepsilon \rightarrow 0}\left\|v_{\varepsilon}-z_{\varepsilon}\right\|_{L^{2}(\Omega)} \leq \delta .
$$

Then $v_{\varepsilon} \stackrel{2}{\longrightarrow} v$.

Proposition 4.5. Let $v_{\varepsilon}$ be a solution of (4.4). Then

$$
v_{\varepsilon}(x) \stackrel{2}{\longrightarrow} \lambda u_{1}(x) b(y, \lambda) \equiv v(x, y),
$$

where $b$ is a solution of (3.9).

Proof. We use estimate (4.5) to simplify equation (4.4). Let $f \in C_{0}^{\infty}\left(\mathbb{R}^{d}\right)$ be such that $\left\|\tilde{u}_{\varepsilon}-f\right\|_{L^{2}(\Omega)} \leq \delta$ for all $\varepsilon$. The existence of such a function follows from the strong convergence $\tilde{u}_{\varepsilon} \rightarrow u_{1}$ in $L^{2}(\Omega)$. Then estimate (4.5) allows us to replace $\tilde{u}_{\varepsilon}$ by $f$. Next, we consider the function $f_{\varepsilon}(x)$ that coincides with $f$ on $\mathbb{R}^{d} \backslash F_{1}^{\varepsilon}$ and that, on each component of $F_{0}^{\varepsilon}$, is equal to the mean value on this component. Then $\left|f-f_{\varepsilon}\right| \rightarrow 0$ uniformly on $\Omega$, and we can replace $f$ by $f_{\varepsilon}$. For the same reason, we can replace $\lambda_{\varepsilon}$ by $\lambda$. After these simplifications, we obtain the equation

$$
T_{\varepsilon} z_{\varepsilon}-\lambda z_{\varepsilon}=\lambda f_{\varepsilon} .
$$

However, in this case,

$$
z_{\varepsilon}(x)=\lambda f_{\varepsilon}(x) b\left(\varepsilon^{-1} x, \lambda\right)
$$




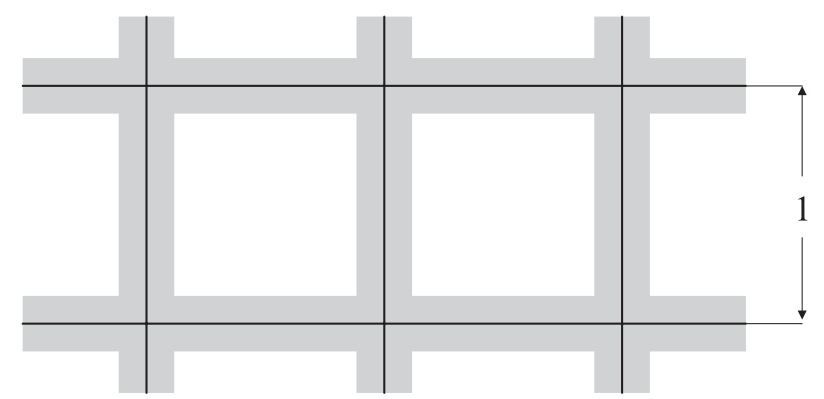

FIGURE 4.

(see (3.9)), and the strong convergence $z_{\varepsilon}(x) \stackrel{2}{\longrightarrow} z(x, y)=\lambda f(x) b(y, \lambda)$ is obvious. As a result, we have

$$
\begin{aligned}
\limsup _{\varepsilon \rightarrow 0}\left\|v_{\varepsilon}-z_{\varepsilon}\right\|_{L^{2}(\Omega)} & \leq C \delta \\
\int_{\Omega} \int_{\square}|v-z|^{2} d x d y & \leq \lambda^{2} \int_{\Omega}\left|u_{1}-f\right|^{2} d x \cdot\left\langle b^{2}\right\rangle \leq C \delta^{2},
\end{aligned}
$$

and Proposition 4.4 gives (4.6). Thus, the compactness lemma is proved.

\section{§5. OTHER GEOMETRIC MODELS}

We describe a simplest plane model in which the rigid phase is not a fixed periodic set as before, but is a "fine" structure with relative area tending to zero.

In Figure 4, we have a square 1-periodic net $F^{h}$ consisting of strips of width $2 h>0$. In the same figure, an infinitely thin (singular) net corresponding to the width $h=0$ is depicted.

On $\mathbb{R}^{2}$, we define a periodic function $\rho^{h}(y)$ such that

$$
2 \rho^{h}(y)= \begin{cases}1+\frac{1}{\left|\square \cap F^{h}\right|} & \text { on } \square \cap F^{h}, \\ 1 & \text { off } F^{h} .\end{cases}
$$

As $h \rightarrow 0$, we have

$$
\rho^{h} d y \rightarrow d \mu, \quad d \mu=\frac{1}{2} d y+\frac{1}{2} d m,
$$

in the sense of weak convergence of measures, where $d y$ is the planar Lebesgue measure and $d m$ is the periodic measure concentrated on the singular net and proportional to the one-dimensional Lebesgue measure on this net, $\int_{\square} d m=1$. It can be said that we "reinforce" the plane with a thin net the mass of which is half the total mass. In the limit as $h \rightarrow 0$, the plane is reinforced with the singular net, which is also half the total mass. The limit measure $\mu$ can be called the composite or junction measure.

Now, we assume that $h(\varepsilon) \rightarrow 0$ and define an $\varepsilon$-periodic thin structure $F^{\varepsilon}$ and the corresponding density $\rho_{\varepsilon}(x)$ by the formulas

$$
F^{\varepsilon}=\varepsilon F^{h(\varepsilon)}, \quad \rho_{\varepsilon}(x)=\rho^{h(\varepsilon)}\left(\varepsilon^{-1} x\right) .
$$

By construction,

$$
2 \rho_{\varepsilon}(x)= \begin{cases}1 & \text { off } F^{\varepsilon}, \\ 1+\frac{1}{\left|\square_{\varepsilon} \cap F_{\varepsilon}\right|} & \text { on } \square_{\varepsilon} \cap F^{\varepsilon},\end{cases}
$$

where $\square_{\varepsilon}=\varepsilon \square$. Obviously, we have $\int_{\varepsilon \square} \rho_{\varepsilon} d x=\varepsilon^{2}$, whence $\rho_{\varepsilon} d x \rightarrow d x$. 


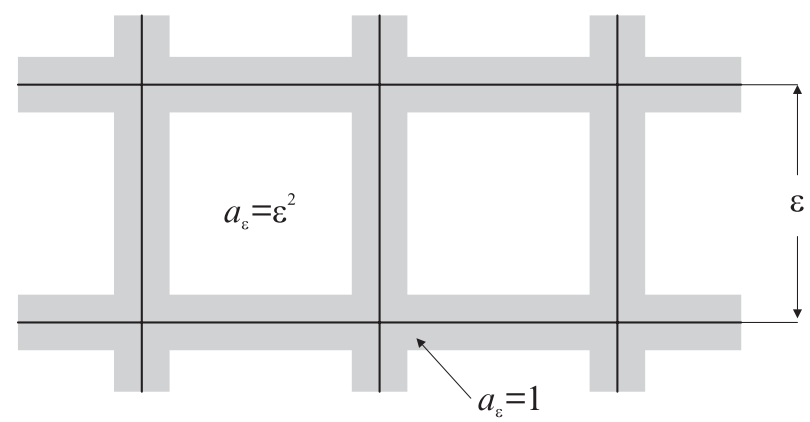

FIGURE 5.

We define the permeability ratio as

$$
a_{\varepsilon}(x)= \begin{cases}1 & \text { on } F^{\varepsilon} \text { (rigid phase) } \\ \varepsilon^{2} & \text { on } \mathbb{R}^{2} \backslash F^{\varepsilon}=F_{0}^{\varepsilon} \text { (soft phase) }\end{cases}
$$

(see Figure 5) and study the spectrum of the operator

$$
A_{\varepsilon}=-\operatorname{div}\left(a_{\varepsilon} \rho_{\varepsilon} \nabla\right)
$$

in the space $L^{2}\left(\mathbb{R}^{d}, \rho_{\varepsilon} d x\right)$. The resolvent equation has the form

$$
-\operatorname{div}\left(a_{\varepsilon} \rho_{\varepsilon} \nabla u_{\varepsilon}\right)+\rho_{\varepsilon} u_{\varepsilon}=f, \quad f \in L^{2}\left(\mathbb{R}^{d}, \rho_{\varepsilon} d x\right) .
$$

First, we introduce an appropriate definition of the two-scale convergence. Let $v_{\varepsilon}$ be a bounded sequence in $L^{2}\left(\mathbb{R}^{d}, \rho_{\varepsilon} d x\right)$, i.e.,

$$
\limsup _{\varepsilon \rightarrow 0} \int_{\mathbb{R}^{d}}\left|v_{\varepsilon}\right|^{2} \rho_{\varepsilon} d x<\infty .
$$

A function $v \in X=L^{2}\left(\mathbb{R}^{d} \times \square, d x \times d \mu\right)=L^{2}\left(\mathbb{R}^{d}, L^{2}(\square, d \mu)\right)$ is the weak two-scale limit of $v_{\varepsilon}, v_{\varepsilon}(x) \stackrel{2}{\rightarrow} v(x, y)$ if

$$
\begin{aligned}
& \lim _{\varepsilon \rightarrow 0} \int_{\mathbb{R}^{d}} v_{\varepsilon}(x) \varphi(x) b\left(\frac{x}{\varepsilon}\right) \rho_{\varepsilon}(x) d x \\
& \quad=\int_{\mathbb{R}^{d}} \int_{\square} v(x, y) \varphi(x) b(y) d x d \mu(y), \quad \varphi \in C_{0}^{\infty}\left(\mathbb{R}^{d}\right), \quad b \in C_{\text {per }}^{\infty}(\square) .
\end{aligned}
$$

In a similar way, we can define strong two-scale convergence. The definitions given in $\S 2$ correspond to the case where $\rho_{\varepsilon} \equiv 1$ and $d \mu=d y$. All properties listed there remain valid also in the general case (see [15], where the general idea of two-scaled convergence was presented). For example, the lower semicontinuity property (2.3) looks like this:

$$
\liminf _{\varepsilon \rightarrow 0} \int_{\mathbb{R}^{d}}\left|v_{\varepsilon}\right|^{2} \rho_{\varepsilon} d x \geq \int_{\mathbb{R}^{d}} \int_{\square}|v|^{2} d x d \mu .
$$

Now, we make a remark concerning the space $X$ to which the two-scale limits belong. The space $L^{2}\left(\mathbb{R}^{d}\right)$ is naturally identified with a subspace of $X$, and since $2 d \mu=d m+d y$ and $d m$ is singular with respect to the Lebesgue measure $d y$, we see that the space $L^{2}\left(\mathbb{R}^{d}, L^{2}(\square, d y)\right)$ can also be regarded as a subspace of $X$.

It can be shown that we have the strong two-scale convergence $A_{\varepsilon} \stackrel{2}{\rightarrow} A$. The limit operator $A$ acts in the subspace $H \subset X$,

$$
H=L^{2}\left(\mathbb{R}^{d}\right)+L^{2}\left(\mathbb{R}^{d}, L^{2}(\square, d y)\right),
$$




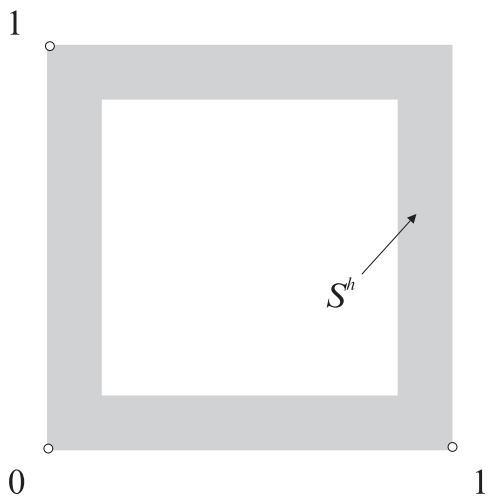

FiguRE 6 .

which is closed because of an inequality of the form (3.2), namely, if $f(x, y)=f_{1}(x)+$ $f_{0}(x, y)$, then

$$
\|f\|_{X}^{2} \geq\left(1-\frac{\sqrt{2}}{2}\right)\left[\int_{\mathbb{R}^{d}}\left|f_{1}\right|^{2} d x+\int_{\square} \int_{\mathbb{R}^{d}}\left|f_{0}\right|^{2} d x d y\right] .
$$

In this case, the homogenized matrix defined by

$$
2 \mathbf{a}^{\text {hom }} \xi \cdot \xi=\inf _{w \in C_{\mathrm{per}}^{\infty}(\square)} \int_{\square}|\xi+\nabla w|^{2} d m
$$

can be calculated explicitly: $\mathbf{a}^{\text {hom }}=\frac{1}{4} I$. The quadratic form $Q(u, u)=(A u, u)$ is given on the set $V=H^{1}\left(\mathbb{R}^{d}\right)+L^{2}\left(\mathbb{R}^{d}, H_{0}^{1}(\square)\right)$ by the formula

$$
\begin{aligned}
Q(u, u) & =\int_{\mathbb{R}^{d}} \mathbf{a}^{\text {hom }} \nabla u_{1} \cdot \nabla \bar{u}_{1} d x+\int_{\square} \int_{\mathbb{R}^{d}} \nabla_{y} u_{0} \cdot \nabla_{y} \bar{u}_{0} d x d \mu \\
& =\frac{1}{4} \int_{\mathbb{R}^{d}} \nabla u_{1} \cdot \nabla \bar{u}_{1} d x+\frac{1}{2} \int_{\square} \int_{\mathbb{R}^{d}} \nabla_{y} u_{0} \cdot \nabla_{y} \bar{u}_{0} d x d y
\end{aligned}
$$

and the relation $A u=P f$, where $f \in X$, reduces to the following two relations:

$$
\begin{cases}-\frac{1}{4} \triangle u_{1}=\langle f\rangle & \text { in } \mathbb{R}^{d},\langle f\rangle=\int_{\square} f d \mu, \\ -\triangle_{y} u_{0}=f & \text { in } \mathbb{R}^{d} \times \square .\end{cases}
$$

Now the operator $A_{0}$ is the Laplace-Dirichlet operator in the unit square $\square$. Thus, we have a complete analogy with the case where the soft phase is dispersed, and the interior of the square $\square$ plays the role of the inclusion $B$.

In the proof of the compactness lemma, we must use the following extension result. Let $S^{h}$ be the frame of width $h$ shown in Figure 6 . Then any function $u \in H^{1}\left(S^{h}\right)$ can be extended up to a function $\tilde{u} \in H^{1}(\square)$ satisfying the estimate

$$
\int_{\square}|\nabla \tilde{u}|^{2} d x \leq \frac{C}{h} \int_{S^{h}}|\nabla u|^{2} d x, \quad \int_{\square}|\nabla \tilde{u}|^{2} d x \leq \frac{C}{h} \int_{S^{h}}\left(|u|^{2}+|\nabla u|^{2}\right) d x,
$$

where the constant $C$ is independent of $u$.

The same results are valid for the three-dimensional model where the thin periodic net is replaced by a thin periodic box structure. The operator $A_{0}$ (by which the spectrum of $A$ and gaps in it are constructed) is the Laplace-Dirichlet operator in the unit cube.

Other net double-porosity models were discussed in [16, 17. 


\section{REFERENCES}

[1] J. D. Joannopoulos, R. D. Meade, and J. N. Winn, Photonic crystals. Molding the flow of light, Princeton Univ. Press, Princeton, NJ, 1995.

[2] A. Figotin and P. Kuchment, Spectral properties of classical waves in high-contrast periodic media, SIAM J. Appl. Math. 58 (1998), 683-702 (electronic). MR1617610(99f:47061)

[3] P. Kuchment and L. Kunyansky, Spectral properties of high contrast band-gap materials and operators on graphs, Experiment. Math. 8 (1999), no. 1, 1-28. MR.1685034(2000c:78027)

[4] Differential operators on graphs and photonic crystals. Modeling and computation in optics and electromagnetics, Adv. Comput. Math. 16 (2002), no. 2-3, 263-290. MR.1892252 (2003h:81270)

[5] V. V. Zhikov, On an extension and an application of the two-scale convergence method, Mat. Sb. 191 (2000), no. 7, 31-72; English transl., Sb. Math. 191 (2000), no. 7-8, 973-1014. MR.1809928 (2001k:35026)

[6] R. Hempel and K. Lienau, Spectral properties of periodic media in the large coupling limit, Comm. Partial Differential Equations 25 (2000), 1445-1470. MR1765136 (2001h:47074)

[7] L. Friedlander, On the density of states of periodic media in the large coupling limit, Preprint ESI no. 1038, Vienna, 2001.

[8] M. Birman and T. Suslina, Threshold effects near the lower edge of the spectrum for periodic differential operators of mathematical physics, Systems, Approximation, Singular Integral Operators, and Related Topics (Bordeaux, 2000), Oper. Theory Adv. Appl., vol. 129, Birkhäuser, Basel, 2001, pp. 71-107. MR 1882692 (2003f:35220)

[9] G. Allaire, Homogenization and two-scale convergence, SIAM J. Math. Anal. 23 (1992), 1482-1518. MR:1185639 (93k:35022)

[10] J. Albert, Genericity of simple eigenvalues for elliptic PDE's, Proc. Amer. Math. Soc. 48 (1975), 413-418. MR0385934(52:6793)

[11] T. Arbogast, J. Douglas, and U. Hornung, Derivation of the double porosity model of single phase flow via homogenization theory, SIAM J. Math. Anal. 21 (1990), no. 4, 823-836. MR1052874 (91d:76074)

[12] U. Hornung (ed.), Homogenization and porous media, Interdisciplinary Appl. Math., vol. 6, Springer-Verlag, New York, 1997. MR1434315 (98h:76128)

[13] G. V. Sandrakov, Averaging of nonstationary equations with contrast coefficients, Dokl. Akad. Nauk 355 (1997), no. 5, 605-608. (Russian) MR1600386 (99a:35018)

[14] V. V. Zhikov, S. M. Kozlov, and O. A. Oleǐnik, Homogenization of differential operators, "Nauka", Moscow, 1993; English transl., Homogenization of differential operators and integral functionals, Springer-Verlag, Berlin, 1994. MR.1318242 (96h:35003a) MR.1329546 (96h:35003b)

[15] V. V. Zhikov, Averaging of problems in the theory of elasticity on singular structures, Izv. Ross. Akad. Nauk Ser. Mat. 66 (2002), no. 2, 81-148; English transl., Izv. Math. 66 (2002), no. 2, 299-365. MR 1918845 (2003f:35024)

[16] A. A. Kotel'nikova and V. V. Zhikov, Spectral aspects in double-porosity models, Abstracts of the International Workshop ANASTHEM, St. Petersburg, Russia, 2000, pp. 38-41.

[17] A. A. Kotel'nikova, On gaps in the spectrum of an elliptic operator on a periodic lattice, Uspekhi Mat. Nauk 59 (2004), 153-154. (Russian) MR2125936

[18] M. Sh. Birman and T. A. Suslina, Second order periodic differential operators. Threshold properties and homogenization, Algebra i Analiz 15 (2003), no. 5, 1-108; English transl., St. Petersburg Math. J. 15 (2004), no. 5, 639-714. MR 2068790

Vladimir State Pedagogical University, Prospekt Stroitelei 11, Vladimir 600024, Russia E-mail address: zhikov@vgpu.vladimir.ru

Received 14/JAN/2004

Translated by B. M. BEKKER 